\title{
ON THE STRETCH FACTOR OF CONVEX DELAUNAY GRAPHS
}

\author{
Prosenjit Bose, ${ }^{*}$ Paz Carmi,${ }^{\dagger}$ Sébastien Collette, ${ }^{\ddagger}$ and Michiel Smid*
}

AbstraCt. Let $C$ be a compact and convex set in the plane that contains the origin in its interior, and let $S$ be a finite set of points in the plane. The Delaunay graph $D G_{C}(S)$ of $S$ is defined to be the dual of the Voronoi diagram of $S$ with respect to the convex distance function defined by $C$. We prove that $D G_{C}(S)$ is a $t$-spanner for $S$, for some constant $t$ that depends only on the shape of the set $C$. Thus, for any two points $p$ and $q$ in $S$, the graph $D G_{C}(S)$ contains a path between $p$ and $q$ whose Euclidean length is at most $t$ times the Euclidean distance between $p$ and $q$.

\section{Introduction}

Let $S$ be a finite set of points in the plane and let $G$ be a graph with vertex set $S$, in which each edge $(p, q)$ has a weight equal to the Euclidean distance $|p q|$ between $p$ and $q$. For a real number $t \geq 1$, we say that $G$ is a $t$-spanner for $S$, if for any two points $p$ and $q$ of $S$, there exists a path in $G$ between $p$ and $q$ whose Euclidean length is at most $t|p q|$. The smallest such $t$ is called the stretch factor of $G$. The problem of constructing spanners has received much attention; see Narasimhan and Smid [12] for an extensive overview.

Spanners were introduced in computational geometry by Chew [3, 4], who proved the following two results. First, the $L_{1}$-Delaunay graph, i.e., the dual of the Voronoi diagram for the Manhattan metric, is a $\sqrt{10}$-spanner. Second, the Delaunay graph based on the convex distance function defined by an equilateral triangle, is a 2-spanner. We remark that in both these results, the stretch factor is measured in the Euclidean metric. Chew also conjectured that the Delaunay graph based on the Euclidean metric, is a $t$-spanner, for some constant $t$. (If not all points of $S$ are on a line, and if no four points of $S$ are cocircular, then the Delaunay graph is the well-known Delaunay triangulation.) This conjecture was proved by Dobkin et al. [8], who showed that $t \leq \pi(1+\sqrt{5}) / 2$. The analysis was improved by Keil and Gutwin [9], who showed that $t \leq \frac{4 \pi \sqrt{3}}{9}$.

In this paper, we unify these results by showing that the Delaunay graph based on any convex distance function has bounded stretch factor. Furthermore, we generalize definitions and results for arbitrary point sets, that is when points are not required to be in general position.

\footnotetext{
${ }^{*}$ School of Computer Science, Carleton University, Ottawa, Ontario, K1S 5B6, Canada. These authors were supported by NSERC.

${ }^{\dagger}$ Department of Computer Science, Ben-Gurion University of the Negev, Beer-Sheva 84105, Israel

${ }^{\ddagger}$ Chargé de recherches du F.R.S.-FNRS. Computer Science Department, Université Libre de Bruxelles, CP212, Bvd du Triomphe, 1050 Brussels, Belgium.
} 
Throughout this paper, we fix a compact and convex set $C$ in the plane. We assume that the origin is in the interior of $C$. A homothet of $C$ is obtained by scaling $C$ with respect to the origin, followed by a translation. Thus, a homothet of $C$ can be written as

$$
x+\lambda C=\{x+\lambda z: z \in C\},
$$

for some point $x$ in the plane and some real number $\lambda \geq 0$. We call $x$ the center of the homothet $x+\lambda C$.

For two points $x$ and $y$ in the plane, we define

$$
d_{C}(x, y):=\min \{\lambda \geq 0: y \in x+\lambda C\} .
$$

If $x \neq y$, then this definition is equivalent to the following: Consider the translate $x+C$ and the ray emanating from $x$ that contains $y$. Let $y^{\prime}$ be the (unique) intersection between this ray and the boundary of $x+C$. Then

$$
d_{C}(x, y)=|x y| /\left|x y^{\prime}\right| .
$$

The function $d_{C}$ is called the convex distance function associated with $C$. Clearly, we have $d_{C}(x, x)=0$ and $d_{C}(x, y)>0$ for all points $x$ and $y$ with $x \neq y$. Chew and Drysdale [5] showed that the triangle inequality $d_{C}(x, z) \leq d_{C}(x, y)+d_{C}(y, z)$ holds. In general, the function $d_{C}$ is not symmetric, i.e., $d_{C}(x, y)$ is not necessarily equal to $d_{C}(y, x)$. If $C$ is symmetric with respect to the origin, however, then $d_{C}$ is symmetric.

Let $S$ be a finite set of points in the plane. For each point $p$ in $S$, we define

$$
V_{C}^{\prime}(p):=\left\{x \in \mathbb{R}^{2}: \text { for all } q \in S, d_{C}(x, p) \leq d_{C}(x, q)\right\} .
$$

If $C$ is not strictly convex ${ }^{1}$, then the set $V_{C}^{\prime}(p)$ may consist of a closed region of positive area with an infinite ray attached to it. For example, in Figure 1, the set $V_{C}^{\prime}(a)$ consists of the set of all points that are on or to the left of the leftmost zig-zag line, together with the infinite horizontal ray that is at the same height as the point $a$. Also, the intersection of two regions $V_{C}^{\prime}(p)$ and $V_{C}^{\prime}(q)$, where $p$ and $q$ are distinct points of $S$, may have a positive area. As a result, the collection $V_{C}^{\prime}(p)$, where $p$ ranges over all points of $S$, does not necessarily give a subdivision of the plane in which the interior of each cell is associated with a unique point of $S$.

In order to obtain such a subdivision, we follow the approach of Klein and Wood [10] (see also Ma [11]): first, infinite rays attached by their apices to regions of positive area are not considered to be part of the region. Second, a point $x$ in $\mathbb{R}^{2}$ that is in the interior of more than one region $V_{C}^{\prime}(p)$ is assigned to the region of the lexicographically smallest point $p$ in $S$ for which $x \in V_{C}^{\prime}(p)$.

To formally define Voronoi cells, let $\prec$ denote the lexicographical ordering on the set of all points in the plane. Let $p_{1} \prec p_{2} \prec \ldots \prec p_{n}$ be the points of $S$, sorted according to this order. Then the Voronoi cells $V_{C}\left(p_{i}\right)$ of the points of $S$ are defined as

$$
V_{C}\left(p_{1}\right):=\operatorname{cl}\left(\operatorname{int}\left(V_{C}^{\prime}\left(p_{1}\right)\right)\right)
$$

\footnotetext{
${ }^{1} C$ is called strictly convex, if for any two points $x$ and $y$ in $C$, the open line segment joining $x$ and $y$ is completely in the interior of $C$.
} 


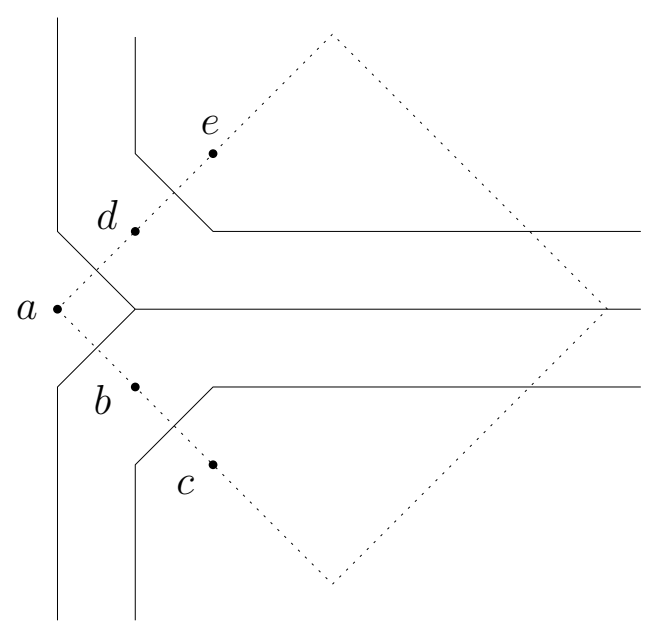

Figure 1: The Voronoi diagram $V D_{C}(S)$ for the set $S=\{a, b, c, d, e\}$. The set $C$ is the square as indicated by the dotted figure; the origin is at the center of $C$.

and, for $1<i \leq n$,

$$
V_{C}\left(p_{i}\right):=\operatorname{cl}\left(\operatorname{int}\left(V_{C}^{\prime}\left(p_{i}\right) \backslash\left(\bigcup_{j<i} V_{C}\left(p_{j}\right)\right)\right)\right),
$$

where $\operatorname{cl}(X)$ and $\operatorname{int}(X)$ denote the closure and the interior of the set $X \subseteq \mathbb{R}^{2}$, respectively.

Thus, in Figure 1, the Voronoi cell $V_{C}(a)$ consists only of the set of all points that are on or to the left of the leftmost zig-zag line; the infinite horizontal ray that is at the same height as the point $a$ is not part of this cell.

The Voronoi diagram $V D_{C}(S)$ of $S$ with respect to $C$ is defined to be the collection of Voronoi cells $V_{C}(p)$, where $p$ ranges over all points of $S$. An example is given in Figure 1.

As for the Euclidean case, the Voronoi diagram $V D_{C}(S)$ induces Voronoi cells, Voronoi edges, and Voronoi vertices. Each point in the plane is either in the interior of a unique Voronoi cell, in the relative interior of a unique Voronoi edge, or a unique Voronoi vertex. Each Voronoi edge $e$ belongs only to the two Voronoi cells that contain $e$ on their boundaries. Observe that Voronoi cells are closed.

The Delaunay graph is defined to be the dual of the Voronoi diagram:

Definition 1 Let $S$ be a finite set of points in the plane. The Delaunay graph $D G_{C}(S)$ of $S$ with respect to $C$ is defined to be the dual of the Voronoi diagram $V_{C}(S)$. That is, the vertex set of $D G_{C}(S)$ is $S$ and two distinct vertices $p$ and $q$ are connected by an edge in $D G_{C}(S)$ if and only if the Voronoi cells $V_{C}(p)$ and $V_{C}(q)$ share a Voronoi edge.

For example, the Delaunay graph $D G_{C}(S)$ for the point set in Figure 1 consists of the five edges $(a, b),(a, d),(b, c),(b, d)$, and $(d, e)$. 

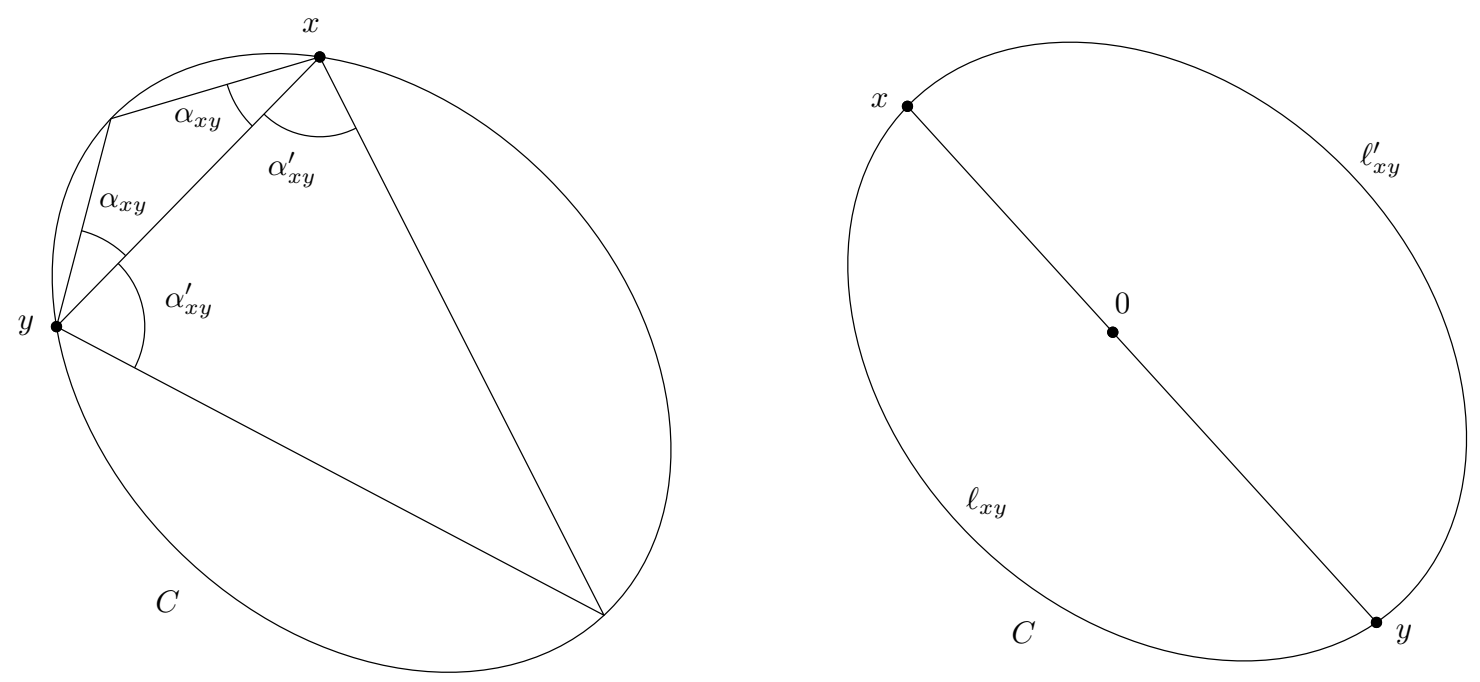

Figure 2: The two parameters associated with $C$.

We consider the Delaunay graph $D G_{C}(S)$ to be a geometric graph, which means that each edge $(p, q)$ is embedded as the closed line segment with endpoints $p$ and $q$.

Before we can state the main result of this paper, we introduce two parameters whose values depend on the shape of the set $C$. Let $x$ and $y$ be two distinct points on the boundary $\partial C$ of $C$. These points partition $\partial C$ into two chains. For each of these chains, there is an isosceles triangle with base $x y$ and whose third vertex is on the chain. Denote the base angles of these two triangles by $\alpha_{x y}$ and $\alpha_{x y}^{\prime}$; see Figure 2 (left). We define

$$
\alpha_{C}:=\min \left\{\max \left(\alpha_{x y}, \alpha_{x y}^{\prime}\right): x, y \in \partial C, x \neq y\right\} .
$$

Consider again two distinct points $x$ and $y$ on $\partial C$, but now assume that $x, y$, and the origin are collinear. As before, $x$ and $y$ partition $\partial C$ into two chains. Let $\ell_{x y}$ and $\ell_{x y}^{\prime}$ denote the lengths of these chains; see Figure 2 (right). We define

$$
\kappa_{C, 0}:=\max \left\{\frac{\max \left(\ell_{x y}, \ell_{x y}^{\prime}\right)}{|x y|}: x, y \in \partial C, x \neq y, \text { and } x, y \text {, and } 0 \text { are collinear }\right\} \text {. }
$$

Clearly, the convex distance function $d_{C}$ and, therefore, the Voronoi diagram $\operatorname{VD} C(S)$, depends on the location of the origin in the interior of $C$. Surprisingly, the Delaunay graph $D G_{C}(S)$ does not depend on this location; see Ma [11, Section 2.1.6]. We define

$$
\kappa_{C}:=\min \left\{\kappa_{C, 0}: 0 \text { is in the interior of } C\right\} .
$$

In this paper, we will prove the following result:

Theorem 1 Let $C$ be a compact and convex set in the plane with a non-empty interior, and let $S$ be a finite set of points in the plane. The stretch factor of the Delaunay graph 
$D G_{C}(S)$ is less than or equal to

$$
t_{C}:= \begin{cases}2 \kappa_{C} \cdot \max \left(\frac{3}{\sin \left(\alpha_{C} / 2\right)}, \kappa_{C}\right) & \text { if } D G_{C}(S) \text { is a triangulation } \\ 2 \kappa_{C}^{2} \cdot \max \left(\frac{3}{\sin \left(\alpha_{C} / 2\right)}, \kappa_{C}\right) & \text { otherwise. }\end{cases}
$$

We emphasize that we do not make any "general position" assumption; our proof of Theorem 1 is valid for any finite set of points in the plane.

Throughout the rest of this paper, we assume that the origin is chosen in the interior of $C$ such that $\kappa_{C}=\kappa_{C, 0}$.

The rest of this paper is organized as follows. In Section 2, we prove some basic properties of the Delaunay graph which are needed in the proof of Theorem 1. In particular, we give a formal proof of the fact that this graph is plane. Even though this fact seems to be well known, we have not been able to find a proof in the literature. Section 3 contains a proof of Theorem 1. This proof is obtained by showing that the Delaunay graph satisfies the "diamond property" and a variant of the "good polygon property" of Das and Joseph [6]. The proof of the latter property is obtained by generalizing the analysis of Dobkin et al. [8] for the lengths of so-called one-sided paths.

\section{Some properties of the Delaunay graph}

Recall that in the Euclidean Delaunay graph, if two points $p$ and $q$ of $S$ are connected by an edge, then there exists a disk having $p$ and $q$ on its boundary that does not contain any point of $S$ in its interior. The next lemma generalizes this result to the Delaunay graph $D G_{C}(S)$. The proof shows that it holds for degenerate point sets as well.

Lemma 1 Let $p$ and $q$ be two points of $S$ and assume that $(p, q)$ is an edge in the Delaunay graph $D G_{C}(S)$. Then, the following are true.

1. The line segment between $p$ and $q$ does not contain any point of $S \backslash\{p, q\}$.

2. For every point $x$ in $V_{C}(p) \cap V_{C}(q)$, there exists a real number $\lambda>0$ such that

(a) the homothet $x+\lambda C$ contains $p$ and $q$ on its boundary, and

(b) the interior of $x+\lambda C$ does not contain any point of $S$.

Proof. To prove the first claim, assume that the line segment between $p$ and $q$ contains a point of $S \backslash\{p, q\}$. Then it follows from Ma [11, Lemma 2.1.4.2] that $V_{C}(p) \cap V_{C}(q)=\emptyset$. Thus, the Voronoi cells of $p$ and $q$ do not share an edge and, therefore, $(p, q)$ is not an edge in the Delaunay graph. This is a contradiction.

The second claim follows from the definition of $D G_{C}(S)$ as the dual of the Voronoi diagram.

As can be seen in Figure 1, Voronoi cells are, in general, not convex. They are, however, star-shaped from the point defining the cell, as proved by Ma [11, Lemma 2.1.4.7]. 


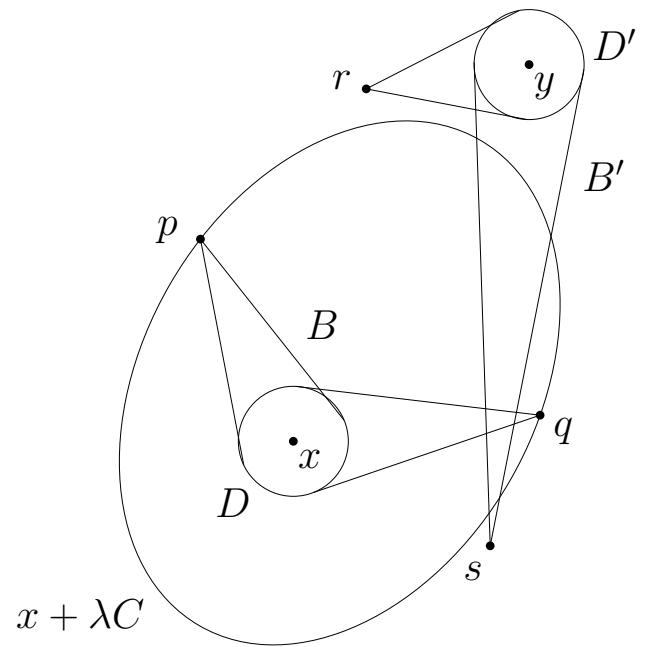

Figure 3: Illustrating the proof of Lemma 2.

It is well known that the Euclidean Delaunay graph is a plane graph; see, for example, de Berg et al. [7, page 189]. The following lemma states that this is true for the Delaunay graph $D G_{C}(S)$ as well.

Lemma 2 The Delaunay graph $D G_{C}(S)$ is a plane graph.

Proof. By the first claim in Lemma $1, D G_{C}(S)$ does not contain two distinct edges $(p, q)$ and $(p, r)$ that are collinear and overlap in a line segment of positive length. Again by the first claim in Lemma $1, D G_{C}(S)$ does not contain two distinct edges $(p, q)$ and $(r, s)$ such that $r$ is on the open line segment joining $p$ and $q$.

It remains to show that $D G_{C}(S)$ does not contain two edges $(p, q)$ and $(r, s)$ that cross properly. The proof is by contradiction. Thus, let $p, q, r$, and $s$ be four pairwise distinct points of $S$, no three of which are collinear, and assume that the line segments $(p, q)$ and $(r, s)$ are edges of $D G_{C}(S)$ that have exactly one point in common.

Since $(p, q)$ is an edge of $D G_{C}(S)$, there exists a point $x$ in the relative interior of $V_{C}(p) \cap V_{C}(q)$. Thus, by the second claim in Lemma 1, there exists a real number $\lambda>0$, such that the homothet $x+\lambda C$ contains $p$ and $q$ on its boundary and no point of $S$ is in the interior of this homothet. Observe that $x$ is in the interior of $x+\lambda C$. Let $D$ be a Euclidean disk centered at $x$ that is contained in the interior of $x+\lambda C$ and that is contained in $V_{C}(p) \cup V_{C}(q)$. We define $B$ to be the set of all 2-link polygonal chains $(p, z, q)$, with $z \in D$; see Figure 3. Observe that $B$ has a positive area. Since $V_{C}(p)$ and $V_{C}(q)$ are star-shaped from $p$ and $q$, respectively, (by [11, Lemma 2.1.4.7]), we have $B \subseteq V_{C}(p) \cup V_{C}(q)$. Since $x+\lambda C$ is convex, we have $B \subseteq x+\lambda C$; in fact, the convex hull of $B$ is contained in $x+\lambda C$. Thus, neither $r$ nor $s$ is in the interior of the convex hull of $B$. Since $p q$ and $r s$ intersect in a point, the line segment $r s$ crosses the set $B$. 


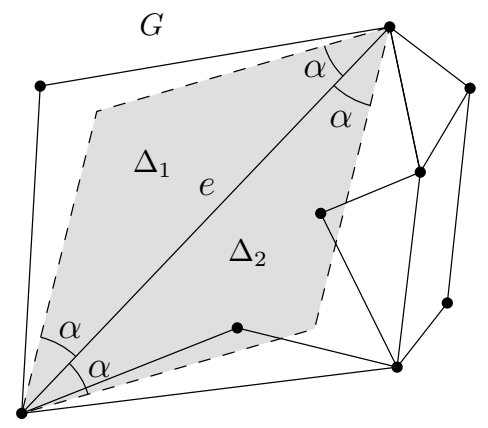

Figure 4: The $\alpha$-diamond property.

By a symmetric argument, since $(r, s)$ is an edge of $D G_{C}(S)$, there exist a point $y$ in the relative interior of $V_{C}(r) \cap V_{C}(s)$ and a real number $\mu>0$, such that $y+\mu C$ contains $r$ and $s$ on its boundary and no point of $S$ is in the interior of this homothet. Let $D^{\prime}$ be a Euclidean disk centered at $y$ that is contained in the interior of $y+\mu C$ and that is contained in $V_{C}(r) \cup V_{C}(s)$. We define $B^{\prime}$ to be the set of all 2-link polygonal chains $(r, z, s)$, with $z \in D^{\prime}$. The set $B^{\prime}$ has a positive area, the line segment $p q$ crosses this set, $B^{\prime} \subseteq V_{C}(r) \cup V_{C}(s)$, and neither $p$ nor $q$ is in the interior of the convex hull of $B^{\prime}$.

It follows that $B$ and $B^{\prime}$ overlap in a region of positive area. Since $B \subseteq V_{C}(p) \cup V_{C}(q)$ and $B^{\prime} \subseteq V_{C}(r) \cup V_{C}(s)$, however, the area of the intersection $B \cap B^{\prime}$ is equal to zero. This is a contradiction. It follows that the edges $(p, q)$ and $(r, s)$ do not cross.

\section{The stretch factor of Delaunay graphs}

In this section, we will prove Theorem 1. First, we show that the Delaunay graph $D G_{C}(S)$ satisfies the diamond property and a variant of the good polygon property of Das and Joseph [6]. According to the results of Das and Joseph, this immediately implies that the stretch factor of $D G_{C}(S)$ is bounded. In fact, we will obtain an upper bound on the stretch factor which is better than the one that is implied by Das and Joseph's result.

\subsection{The diamond property}

Let $G$ be a plane graph with vertex set $S$ and let $\alpha$ be a real number with $0<\alpha<\pi / 2$. For any edge $e$ of $G$, let $\Delta_{1}$ and $\Delta_{2}$ be the two isosceles triangles with base $e$ and base angle $\alpha$; see Figure 4. We say that $e$ satisfies the $\alpha$-diamond property, if at least one of the triangles $\Delta_{1}$ and $\Delta_{2}$ does not contain any point of $S$ in its interior. The graph $G$ is said to satisfy the $\alpha$-diamond property, if every edge $e$ of $G$ satisfies this property.

Lemma 3 Consider the value $\alpha_{C}$ that was defined in Section 1. The Delaunay graph $D G_{C}(S)$ satisfies the $\alpha_{C}$-diamond property. 


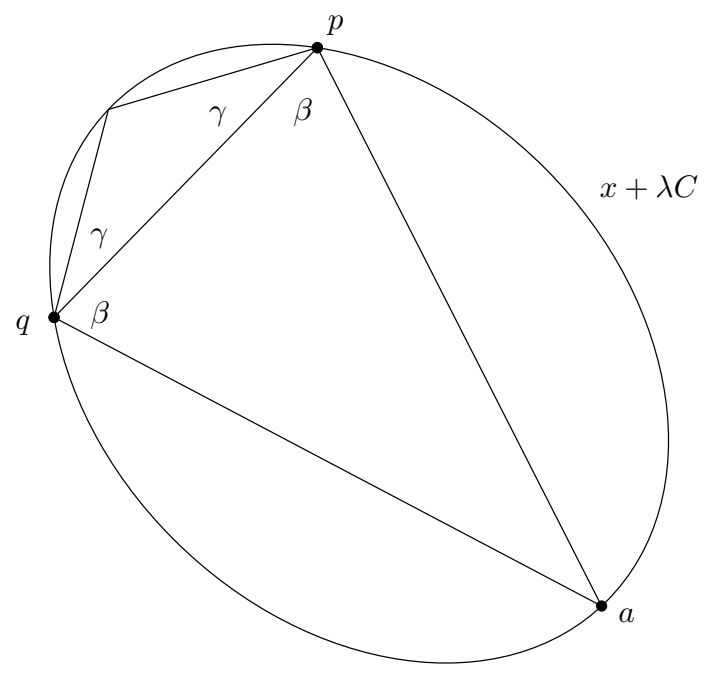

Figure 5: Illustrating the proof of Lemma 3.

Proof. Let $(p, q)$ be an arbitrary edge of $D G_{C}(S)$ and let $x$ be any point in the relative interior of $V_{C}(p) \cap V_{C}(q)$. By Lemma 1, there exists a real number $\lambda>0$ such that $p$ and $q$ are on the boundary of the homothet $x+\lambda C$ and no point of $S$ is in the interior of $x+\lambda C$. The points $p$ and $q$ partition $\partial(x+\lambda C)$ into two chains. For each of these chains, there is an isosceles triangle with base $p q$ and whose third vertex is on the chain. We denote the base angles of these two triangles by $\beta$ and $\gamma$; see Figure 5 . We may assume without loss of generality that $\beta \geq \gamma$. Let $a$ denote the third vertex of the triangle with base angle $\beta$. If we translate $x+\lambda C$ so that $x$ coincides with the origin and scale the translated homothet by a factor of $1 / \lambda$, then we obtain the set $C$. This translation and scaling does not change the angles $\beta$ and $\gamma$. Thus, using the notation of Section 1 (see also Figure 2), we have $\{\beta, \gamma\}=\left\{\alpha_{p q}, \alpha_{p q}^{\prime}\right\}$. The definition of $\alpha_{C}$ then implies that

$$
\alpha_{C} \leq \max \left(\alpha_{p q}, \alpha_{p q}^{\prime}\right)=\beta
$$

Let $\Delta$ be the isosceles triangle with base $p q$ and base angle $\alpha_{C}$ such that $a$ and the third vertex of $\Delta$ are on the same side of $p q$. Then $\Delta$ is contained in the triangle with vertices $p$, $q$, and $a$. Since the latter triangle is contained in $x+\lambda C$, it does not contain any point of $S$ in its interior. Thus, $\Delta$ does not contain any point of $S$ in its interior. This proves that the edge $(p, q)$ satisfies the $\alpha_{C}$-diamond property.

\subsection{The visible-pair spanner property}

For a real number $\kappa \geq 1$, we say that the plane graph $G$ satisfies the strong visible-pair $\kappa$-spanner property, if the following is true: For every face $f$ of $G$, and for every two vertices $p$ and $q$ on the boundary of $f$, such that the open line segment joining $p$ and $q$ is completely in the interior of $f$, the graph $G$ contains a path between $p$ and $q$ having length at most 
$\kappa|p q|$. If for every face $f$ of $G$ and for every two vertices $p$ and $q$ on the boundary of $f$, such that the line segment $p q$ does not intersect the exterior of $f$, the graph $G$ contains a path between $p$ and $q$ having length at most $\kappa|p q|$, then we say that $G$ satisfies the visible-pair $\kappa$-spanner property. Observe that the former property implies the latter one. Also, observe that these properties are variants of the $\kappa$-good polygon property of Das and Joseph [6]: The $\kappa$-good polygon property requires that $G$ contains a path between $p$ and $q$ that is along the boundary of $f$ and whose length is at most $\kappa|p q|$; in the (strong) visible-pair spanner property, the path is not required to be along the boundary of $f$.

In this subsection, we will prove that the Delaunay graph $D G_{C}(S)$ satisfies the visible-pair $\kappa_{C}$-spanner property, where $\kappa_{C}$ is as defined in Section 1 . This claim will be proved by generalizing results of Dobkin et al. [8] on so-called one-sided paths.

Let $p$ and $q$ be two distinct points of $S$ and assume that $(p, q)$ is not an edge of the Delaunay graph $D G_{C}(S)$. Consider the Voronoi diagram $V D_{C}(S)$. We consider the sequence of points in $S$ whose Voronoi cells are visited when the line segment $p q$ is traversed from $p$ to $q$. If $p q$ does not contain any Voronoi vertex, then this sequence forms a path in $D G_{C}(S)$ between $p$ and $q$. Since, in general, Voronoi cells are not convex, it may happen that this path contains duplicates. In order to avoid this, we define the sequence in the following way.

In the rest of this section, we will refer to the line through $p$ and $q$ as the $X$-axis, and we will say that $p$ is to the left of $q$. This implies a left-to-right order on the $X$-axis, the notion of a point being above or below the X-axis, as well as the notions horizontal and vertical. (Thus, conceptually, we rotate and translate all points of $S$, the set $C$, the Voronoi diagram $V D_{C}(S)$, and the $D G_{C}(S)$, such that $p$ and $q$ are on a horizontal line and $p$ is to the left of $q$. Observe that $V D_{C}(S)$ is still defined based on the lexicographical order of the points of $\mathrm{S}$ before this rotation and translation.) In the following, we consider the (horizontal) line segment $p q$. If this segment contains a Voronoi vertex, then we imagine moving $p q$ vertically upwards by an infinitesimal amount. Thus, we may assume that $p q$ does not contain any Voronoi vertex of the (rotated and translated) Voronoi diagram $V D_{C}(S)$.

The first point in the sequence is $p_{0}:=p$. We define $x_{1} \in \mathbb{R}^{2}$ to be the point on the line segment $p q$ such that $x_{1} \in V_{C}\left(p_{0}\right)$ and $x_{1}$ is closest to $q$.

Let $i \geq 1$ and assume that the points $p_{0}, p_{1}, \ldots, p_{i-1}$ of $S$ and the points $x_{1}, \ldots, x_{i}$ in $\mathbb{R}^{2}$ have already been defined, where $x_{i}$ is the point on the line segment $p q$ such that $x_{i} \in V_{C}\left(p_{i-1}\right)$ and $x_{i}$ is closest to $q$. If $p_{i-1}=q$, then the construction is completed. Otherwise, observe that $x_{i}$ is in the relative interior of a Voronoi edge. We define $p_{i}$ to be the point of $S \backslash\left\{p_{i-1}\right\}$ whose Voronoi cell contains $x_{i}$ on its boundary, and define $x_{i+1}$ to be the point on the line segment $p q$ such that $x_{i+1} \in V_{C}\left(p_{i}\right)$ and $x_{i+1}$ is closest to $q$.

Let $p=p_{0}, p_{1}, \ldots, p_{k}=q$ be the sequence of points in $S$ obtained in this way. By construction, these $k+1$ points are pairwise distinct and for each $i$ with $1 \leq i \leq k$, the Voronoi cells $V_{C}\left(p_{i-1}\right)$ and $V_{C}\left(p_{i}\right)$ share an edge. Therefore, by definition, $\left(p_{i-1}, p_{i}\right)$ is an edge in $D G_{C}(S)$. Thus, $p=p_{0}, p_{1}, \ldots, p_{k}=q$ defines a path in $D G_{C}(S)$ between $p$ and $q$. We call this path the direct path between $p$ and $q$. If all points $p_{1}, p_{2}, \ldots, p_{k-1}$ are strictly on one side of the line through $p$ and $q$, then we say that the direct path is one-sided. 

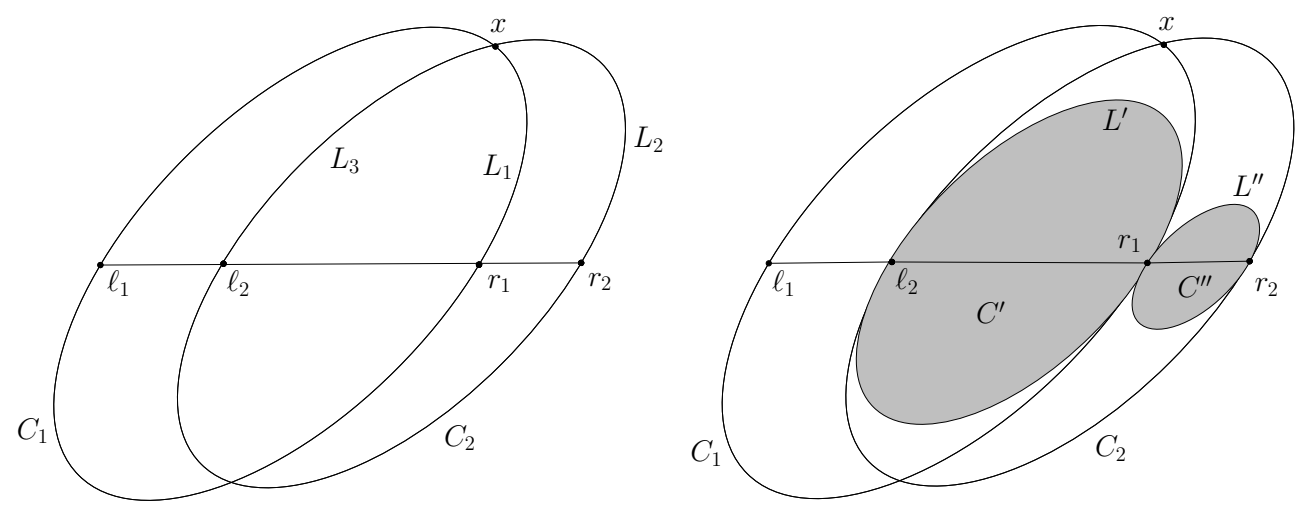

Figure 6: Illustrating the proof of Lemma 4.

We will show in Lemma 5 that the length of a one-sided path is at most $\kappa_{C}|p q|$. The proof of this lemma uses a geometric property which we prove first.

Let $C^{\prime}$ be a homothet of $C$ whose center is on the $X$-axis, and let $x$ and $y$ be two points on the boundary of $C^{\prime}$ that are on or above the $X$-axis. The points $x$ and $y$ partition the boundary of $C^{\prime}$ into two chains. One of these chains is completely on or above the $X$-axis; we denote this chain by $\operatorname{arc}\left(x, y ; C^{\prime}\right)$. The length of this chain is denoted by $\left|\operatorname{arc}\left(x, y ; C^{\prime}\right)\right|$.

For two points $x$ and $y$ on the $X$-axis, we write $x<_{X} y$ if $x$ is strictly to the left of $y$, and we write $x \leq_{X} y$ if $x=y$ or $x<_{X} y$.

We now state the geometric property, which is illustrated in Figure 6. Recall the value $\kappa_{C}$ that was defined in Section 1.

Lemma 4 Let $C_{1}=y_{1}+\lambda_{1} C$ and $C_{2}=y_{2}+\lambda_{2} C$ be two homothets of $C$ whose centers $y_{1}$ and $y_{2}$ are on the $X$-axis. Assume that $\lambda_{1}>0, \lambda_{2}>0$, and $y_{1}<_{X} y_{2}$. For $i=1,2$, let $\ell_{i}$ and $r_{i}$ be the leftmost and rightmost points of $C_{i}$ on the $X$-axis, respectively. Assume that $r_{1} \leq_{X} r_{2}$ and $\ell_{1} \leq_{X} \ell_{2}<_{X} r_{1}$. Let $x$ be a point that is on the boundaries of both $C_{1}$ and $C_{2}$ and on or above the $X$-axis. Let $L_{1}=\left|\operatorname{arc}\left(x, r_{1} ; C_{1}\right)\right|$ and $L_{2}=\left|\operatorname{arc}\left(x, r_{2} ; C_{2}\right)\right|$. Then

$$
L_{2} \leq L_{1}+\kappa_{C}\left|r_{1} r_{2}\right|
$$

Proof. We define $L_{3}=\left|\operatorname{arc}\left(\ell_{2}, x ; C_{2}\right)\right|$. Let $C^{\prime}$ be the homothet of $C$ whose center is on the $X$-axis such that the intersection between $C^{\prime}$ and the $X$-axis is equal to the line segment $\ell_{2} r_{1}$, and let $L^{\prime}=\left|\operatorname{arc}\left(\ell_{2}, r_{1} ; C^{\prime}\right)\right|$; see Figure 6. Observe that, for $\lambda:=\left|\ell_{2} r_{1}\right| /\left|\ell_{2} r_{2}\right|, C^{\prime}$ is obtained from $C_{2}$ by a scaling by a factor of $\lambda$. Thus, since $\left|\operatorname{arc}\left(\ell_{2}, r_{2} ; C_{2}\right)\right|=L_{2}+L_{3}$, we have

$$
L^{\prime}=\lambda\left(L_{2}+L_{3}\right)
$$

Let $C^{\prime \prime}$ be the homothet of $C$ whose center is on the $X$-axis such that the intersection between $C^{\prime \prime}$ and the $X$-axis is equal to the line segment $r_{1} r_{2}$, and let $L^{\prime \prime}=\left|\operatorname{arc}\left(r_{1}, r_{2} ; C^{\prime \prime}\right)\right|$. 
Since $C^{\prime \prime}$ is obtained from $C_{2}$ by a scaling by a factor of $1-\lambda$, we have

$$
L^{\prime \prime}=(1-\lambda)\left(L_{2}+L_{3}\right)
$$

Thus, we have

$$
L^{\prime}+L^{\prime \prime}=L_{2}+L_{3}
$$

By convexity, we have $C^{\prime} \subseteq C_{1} \cap C_{2}$. Then it follows, again from convexity (see Benson $[1$, page 42]), that

$$
L^{\prime} \leq L_{1}+L_{3}
$$

Thus, we have

$$
L_{2}+L_{3}=L^{\prime}+L^{\prime \prime} \leq L_{1}+L_{3}+L^{\prime \prime}
$$

which implies that

$$
L_{2} \leq L_{1}+L^{\prime \prime}
$$

Since, by the definition of $\kappa_{C}, L^{\prime \prime} \leq \kappa_{C}\left|r_{1} r_{2}\right|$, the proof is complete.

We are now ready to prove an upper bound on the length of a one-sided path.

Lemma 5 If the direct path between $p$ and $q$ is one-sided, then its length is at most $\kappa_{C}|p q|$.

Proof. As above, we assume that $p$ and $q$ are on the $X$-axis and that $p$ is to the left of $q$. Consider the direct path $p=p_{0}, p_{1}, \ldots, p_{k}=q$ in $D G_{C}(S)$ and the sequence $x_{1}, x_{2}, \ldots, x_{k}$, as defined above. Since the direct path is one-sided, we may assume without loss of generality that the points $p_{1}, p_{2}, \ldots, p_{k-1}$ are strictly above the $X$-axis. We have to show that

$$
\sum_{i=1}^{k}\left|p_{i-1} p_{i}\right| \leq \kappa_{C}|p q| .
$$

Recall that, for each $i$ with $1 \leq i \leq k, x_{i}$ is in the relative interior of $V_{C}\left(p_{i-1}\right) \cap V_{C}\left(p_{i}\right)$ and $x_{i}$ is on the line segment $p q$. Therefore, by Lemma 1 , if we define $\lambda_{i}:=d_{C}\left(x_{i}, p_{i-1}\right)$ (which is equal to $d_{C}\left(x_{i}, p_{i}\right)$ ), then the homothet $C_{i}:=x_{i}+\lambda_{i} C$ contains $p_{i-1}$ and $p_{i}$ on its boundary and no point of $S$ is in its interior.

For each $i$ with $1 \leq i \leq k$, let $\ell_{i}$ and $r_{i}$ be the leftmost and rightmost points of $C_{i}$ that are on the $X$-axis, respectively. We will prove that for each $j$ with $1 \leq j \leq k$,

$$
\sum_{i=1}^{j-1}\left|p_{i-1} p_{i}\right|+\left|\operatorname{arc}\left(p_{j-1}, r_{j} ; C_{j}\right)\right| \leq \kappa_{C}\left|p r_{j}\right| .
$$

For $j=k$, inequality (2) implies (1), because $r_{k}=p_{k}=q$.

Before we prove (2), we show that $\ell_{1} \leq_{X} \ell_{2} \leq_{X} \ldots \leq_{X} \ell_{k}$. Observe that $x_{1}<_{X}$ $x_{2}<_{X} \ldots<_{X} x_{k}$. Assume that there is an index $i$ such that $\ell_{i}<_{X} \ell_{i-1}$. Since $\ell_{i}<_{X}$ $\ell_{i-1}<_{X} x_{i-1}<_{X} x_{i}$, it follows that $\lambda_{i-1}<\lambda_{i}$. If $r_{i-1}<_{X} r_{i}$, then $\ell_{i}<_{X} \ell_{i-1}<_{X} r_{i-1}<_{X} r_{i}$ and, therefore, $C_{i-1}$ is completely contained in the interior of $C_{i}$. This is a contradiction, 
because $p_{i-1}$ is on the boundary of $C_{i-1}$, but no point of $S$ is in the interior of $C_{i}$. Thus, we have $r_{i} \leq_{X} r_{i-1}$. Since $x_{i-1}<_{X} x_{i}<_{X} r_{i} \leq_{X} r_{i-1}$, we have $\lambda_{i-1}>\lambda_{i}$, which is a contradiction.

Thus, we have shown that $\ell_{1} \leq_{X} \ell_{2} \leq_{X} \ldots \leq_{X} \ell_{k}$. By a symmetric argument, it follows that $r_{1} \leq_{X} r_{2} \leq_{X} \cdots \leq_{X} r_{k}$.

Now we are ready to prove (2). The proof is by induction on $j$. For the base case, i.e., when $j=1$, we have to show that

$$
\left|\operatorname{arc}\left(p_{0}, r_{1} ; C_{1}\right)\right| \leq \kappa_{C}\left|p r_{1}\right| .
$$

Since $p_{0}=p=\ell_{1}$, this inequality follows from the definition of $\kappa_{C}$.

Let $1 \leq j<k$ and assume that (2) holds for $j$. We have to show that (2) holds for $j+1$, i.e.,

$$
\sum_{i=1}^{j}\left|p_{i-1} p_{i}\right|+\left|\operatorname{arc}\left(p_{j}, r_{j+1} ; C_{j+1}\right)\right| \leq \kappa_{C}\left|p r_{j+1}\right| .
$$

By the induction hypothesis, we have

$$
\begin{aligned}
& \sum_{i=1}^{j}\left|p_{i-1} p_{i}\right|+\left|\operatorname{arc}\left(p_{j}, r_{j+1} ; C_{j+1}\right)\right| \\
& \quad=\sum_{i=1}^{j-1}\left|p_{i-1} p_{i}\right|+\left|p_{j-1} p_{j}\right|+\left|\operatorname{arc}\left(p_{j}, r_{j+1} ; C_{j+1}\right)\right| \\
& \quad \leq \kappa_{C}\left|p r_{j}\right|-\left|\operatorname{arc}\left(p_{j-1}, r_{j} ; C_{j}\right)\right|+\left|p_{j-1} p_{j}\right|+\left|\operatorname{arc}\left(p_{j}, r_{j+1} ; C_{j+1}\right)\right| \\
& \quad=\kappa_{C}\left(\left|p r_{j+1}\right|-\left|r_{j} r_{j+1}\right|\right)-\left|\operatorname{arc}\left(p_{j-1}, r_{j} ; C_{j}\right)\right|+\left|p_{j-1} p_{j}\right|+\left|\operatorname{arc}\left(p_{j}, r_{j+1} ; C_{j+1}\right)\right| .
\end{aligned}
$$

Thus, (3) holds if we can show that

$$
\left|p_{j-1} p_{j}\right|+\left|\operatorname{arc}\left(p_{j}, r_{j+1} ; C_{j+1}\right)\right| \leq\left|\operatorname{arc}\left(p_{j-1}, r_{j} ; C_{j}\right)\right|+\kappa_{C}\left|r_{j} r_{j+1}\right| .
$$

We distinguish two cases.

Case 1: $r_{j} \leq_{X} \ell_{j+1}$.

By the triangle inequality, we have

$$
\left|p_{j-1} p_{j}\right| \leq\left|p_{j-1} r_{j}\right|+\left|r_{j} \ell_{j+1}\right|+\left|\ell_{j+1} p_{j}\right| .
$$

Since $p_{j}$ is on the boundary of $C_{j+1}$ and strictly above the $X$-axis, we have

$$
\begin{aligned}
\left|\ell_{j+1} p_{j}\right|+\left|\operatorname{arc}\left(p_{j}, r_{j+1} ; C_{j+1}\right)\right| & \leq\left|\operatorname{arc}\left(\ell_{j+1}, p_{j} ; C_{j+1}\right)\right|+\left|\operatorname{arc}\left(p_{j}, r_{j+1} ; C_{j+1}\right)\right| \\
& =\left|\operatorname{arc}\left(\ell_{j+1}, r_{j+1} ; C_{j+1}\right)\right| \\
& \leq \kappa_{C}\left|\ell_{j+1} r_{j+1}\right| .
\end{aligned}
$$

It follows that

$$
\begin{aligned}
\left|p_{j-1} p_{j}\right|+\left|\operatorname{arc}\left(p_{j}, r_{j+1} ; C_{j+1}\right)\right| & \leq\left|p_{j-1} r_{j}\right|+\left|r_{j} \ell_{j+1}\right|+\kappa_{C}\left|\ell_{j+1} r_{j+1}\right| \\
& \leq\left|\operatorname{arc}\left(p_{j-1}, r_{j} ; C_{j}\right)\right|+\kappa_{C}\left|r_{j} \ell_{j+1}\right|+\kappa_{C}\left|\ell_{j+1} r_{j+1}\right| \\
& =\left|\operatorname{arc}\left(p_{j-1}, r_{j} ; C_{j}\right)\right|+\kappa_{C}\left|r_{j} r_{j+1}\right| .
\end{aligned}
$$


Thus, (4) holds.

Case 2: $\ell_{j+1}<_{X} r_{j}$.

Since $p_{j}$ is on the boundaries of both $C_{j}$ and $C_{j+1}$ and strictly above the $X$-axis, we can apply Lemma 4 with $x=p_{j}$ and obtain

$$
\left|\operatorname{arc}\left(p_{j}, r_{j+1} ; C_{j+1}\right)\right| \leq\left|\operatorname{arc}\left(p_{j}, r_{j} ; C_{j}\right)\right|+\kappa_{C}\left|r_{j} r_{j+1}\right| .
$$

Thus,

$$
\left|p_{j-1} p_{j}\right|+\left|\operatorname{arc}\left(p_{j}, r_{j+1} ; C_{j+1}\right)\right| \leq\left|p_{j-1} p_{j}\right|+\left|\operatorname{arc}\left(p_{j}, r_{j} ; C_{j}\right)\right|+\kappa_{C}\left|r_{j} r_{j+1}\right| .
$$

We claim that $p_{j} \in \operatorname{arc}\left(p_{j-1}, r_{j}, C_{j}\right)$. Assuming this is true, it follows that

$$
\begin{aligned}
\left|p_{j-1} p_{j}\right|+\left|\operatorname{arc}\left(p_{j}, r_{j+1} ; C_{j+1}\right)\right| & \leq\left|\operatorname{arc}\left(p_{j-1}, p_{j} ; C_{j}\right)\right|+\left|\operatorname{arc}\left(p_{j}, r_{j} ; C_{j}\right)\right|+\kappa_{C}\left|r_{j} r_{j+1}\right| \\
& =\left|\operatorname{arc}\left(p_{j-1}, r_{j} ; C_{j}\right)\right|+\kappa_{C}\left|r_{j} r_{j+1}\right|,
\end{aligned}
$$

i.e., (4) holds.

It remains to prove that $p_{j} \in \operatorname{arc}\left(p_{j-1}, r_{j}, C_{j}\right)$. Since $p_{0}=\ell_{0}$ and $p_{1}$ is strictly above the $X$-axis, this is true for $j=1$. Assume that $2 \leq j<k$ and $p_{j} \notin \operatorname{arc}\left(p_{j-1}, r_{j}, C_{j}\right)$. Then, since $p_{j}$ is strictly above the $X$-axis, $p_{j-1}$ is in the relative interior of $\operatorname{arc}\left(p_{j}, r_{j}, C_{j}\right)$.

By the definition of the point $x_{j}$, there is a point $y$ on the $X$-axis such that $y<_{X} x_{j}$ and the line segment $y x_{j}$ is contained in the Voronoi cell $V_{C}\left(p_{j-1}\right)$. Using the additional fact that the cell is star-shaped, the triangle $\Delta$ with vertices $p_{j-1}, y$, and $x_{j}$ is contained in $V_{C}\left(p_{j-1}\right)$.

Again by the definition of the point $x_{j}$, there is a point $z$ on the $X$-axis such that $x_{j}<_{X} z$ and the line segment $x_{j} z$ is contained in the Voronoi cell $V_{C}\left(p_{j}\right)$, which is starshaped. Thus, the triangle $\Delta^{\prime}$ with vertices $p_{j}, x_{j}$, and $z$ is contained in $V_{C}\left(p_{j}\right)$.

Since $p_{j-1}$ and $p_{j}$ are strictly above the $X$-axis and since $p_{j-1}$ is in the relative interior of $\operatorname{arc}\left(p_{j}, r_{j}, C_{j}\right)$, the intersection of $\Delta$ and $\Delta^{\prime}$ has a positive area and is contained in the intersection of $V_{C}\left(p_{j-1}\right)$ and $V_{C}\left(p_{j}\right)$. This is a contradiction, because the area of the intersection of any two Voronoi cells is zero.

We are now ready to prove that the Delaunay graph satisfies the visible-pair spanner property:

Lemma 6 The Delaunay graph $D G_{C}(S)$ satisfies the visible-pair $\kappa_{C}$-spanner property.

Proof. Recall from Lemma 2, that the graph $D G_{C}(S)$ is plane. It suffices to prove that $D G_{C}(S)$ satisfies the strong visible-pair $\kappa_{C}$-spanner property. Let $f$ be a face of $G$ and let $p$ and $q$ be two vertices on $f$ such that the open line segment between $p$ and $q$ is contained in the interior of $f$. We have to show that there is a path in $D G_{C}(S)$ between $p$ and $q$ whose length is at most $\kappa_{C}|p q|$.

As before, we assume that $p$ and $q$ are on the $X$-axis and that $p$ is to the left of $q$. Consider the direct path $p=p_{0}, p_{1}, \ldots, p_{k}=q$ in $D G_{C}(S)$ and the sequence $x_{1}, x_{2}, \ldots, x_{k}$, 
as defined in the beginning of this section. We will show that the direct path is one-sided. The lemma then follows from Lemma 5 .

Since the open line segment between $p$ and $q$ is in the interior of $f$, none of the points $p_{1}, \ldots, p_{k-1}$ is on the closed line segment $p q$. Assume that for some $i$ with $1 \leq i<k$, $p_{i}$ is on the $X$-axis. Then $p_{i}$ is either strictly to the left of $p$ or strictly to the right of $q$. We may assume without loss of generality that $p_{i}$ is strictly to the right of $q$. Consider the point $x_{i}$ and the homothet $C_{i}=x_{i}+\lambda_{i} C$ as in the proof of Lemma 5. Since $x_{i}$ is on $p q$ and in the interior of $C_{i}$, and since $p_{i}$ is on the boundary of $C_{i}$, it follows from convexity that $q$ is in the interior of $C_{i}$, which is a contradiction. Thus we have shown that none of the points $p_{1}, \ldots, p_{k-1}$ is on the $X$-axis.

Assume that the direct path is not one-sided. Then there is an edge $\left(p_{i-1}, p_{i}\right)$ on this path such that one of $p_{i-1}$ and $p_{i}$ is strictly below the $X$-axis and the other point is strictly above the $X$-axis. Let $z$ be the intersection between $p_{i-1} p_{i}$ and the $X$-axis. By assumption, $z$ is not on the open line segment joining $p$ and $q$, and by Lemma $1, z \neq p$ and $z \neq q$. Thus, $z$ is either strictly to the left of $p$ or strictly to the right of $q$. We may assume without loss of generality that $z$ is strictly to the right of $q$. Consider again the point $x_{i}$ and the homothet $C_{i}=x_{i}+\lambda_{i} C$ as in the proof of Lemma 5. This homothet contains the points $x_{i}, p_{i-1}$ and $p_{i}$. Thus, by convexity, $C_{i}$ contains the triangle with vertices $x_{i}, p_{i-1}$, and $p_{i}$. Since $q$ is in the interior of this triangle, it follows that $q$ is in the interior of $C_{i}$, which is a contradiction.

\subsection{The proof of Theorem 1}

Das and Joseph [6] have shown that any plane graph satisfying the diamond property and the good polygon property has a bounded stretch factor. The analysis of the stretch factor was slightly improved by Bose et al. [2]. A close inspection of the proof in [2] shows that the following holds: Let $G$ be a geometric graph with the following four properties:

1. $G$ is plane.

2. $G$ satisfies the $\alpha$-diamond property.

3. The stretch factor of any one-sided path in $G$ is at most $\kappa$.

4. $G$ satisfies the visible-pair $\kappa^{\prime}$-spanner property.

Then, $G$ is a $t$-spanner for

$$
t=2 \kappa \kappa^{\prime} \cdot \max \left(\frac{3}{\sin (\alpha / 2)}, \kappa\right)
$$

We have shown that the Delaunay graph $D G_{C}(S)$ satisfies all these properties: By Lemma 2, $D G_{C}(S)$ is plane. By Lemma $3, D G_{C}(S)$ satisfies the $\alpha_{C}$-diamond property. By Lemma 5 , the stretch factor of any one-sided path in $D G_{C}(S)$ is at most $\kappa_{C}$. By Lemma $6, D G_{C}(S)$ satisfies the visible-pair $\kappa_{C}$-spanner property. If $D G_{C}(S)$ is a triangulation, then obviously, $D G_{C}(S)$ satisfies the visible-pair 1-spanner property. Therefore, we have completed the proof of Theorem 1. 


\section{Concluding remarks}

We have considered the Delaunay graph $D G_{C}(S)$, where $C$ is a compact and convex set with a non-empty interior and $S$ is a finite set of points in the plane. We have shown that the (Euclidean) stretch factor of $D G_{C}(S)$ is bounded from above by a function of two parameters $\alpha_{C}$ and $\kappa_{C}$ that are determined only by the shape of $C$. Roughly speaking, these two parameters give a measure of the "fatness" of the set $C$.

For instance, if $C$ is the disk of radius one in the $L_{p}$-metric, with $1 \leq p \leq \infty$, then $\alpha_{C}=\pi / 4$ and $\kappa_{C}$ ranges from $\pi / 2$ (for $p=2$ ) to 2 (for $p=\infty$ in which case $C$ is an axes-parallel square). Note that $\kappa_{C}=2$ for $p=1$ as well, as the $L_{1}$-disk is the same as the $L_{\infty}$-disk up to an affine transformation. This implies that the stretch factor will be similar for all $L_{p}$-unit disks. For general sets $C$, the stretch factor can, however, be arbitrarily big, for instance when $C$ is a rectangle of infinitesimal width.

Our analysis provides the first generic bound valid for any compact and convex set $C$. In all previous works, only special examples of such sets $C$ were considered. Furthermore, our approach does not make any "general position" assumption about the point set $S$, while most related works on Delaunay graphs do not consider the case when four points are cocircular.

Note that for the Euclidean Delaunay triangulation (i.e., when the set $C$ is the disk of radius one, and with no four cocircular points), we have $\alpha_{C}=\pi / 4$ and $\kappa_{C}=\pi / 2$, and we derive an upper bound on the stretch factor of $\frac{3 \pi}{\sin (\pi / 8)} \approx 24.6$.

Observe that this is much worse than the currently best known upper bound (as proved by Keil and Gutwin [9]), which is $\frac{4 \pi \sqrt{3}}{9} \approx 2.42$. We leave open the problem of improving our upper bound. In particular, is it possible to generalize the techniques of Dobkin et al. [8] and Keil and Gutwin [9], from the Euclidean metric to an arbitrary convex distance function?

\section{References}

[1] R. V. Benson. Euclidean Geometry and Convexity. McGraw-Hill, New York, NY, 1966.

[2] P. Bose, A. Lee, and M. Smid. On generalized diamond spanners. In Proceedings of the 10th Workshop on Algorithms and Data Structures, volume 4619 of Lecture Notes in Computer Science, pages 325-336, Berlin, 2007. Springer-Verlag.

[3] L. P. Chew. There is a planar graph almost as good as the complete graph. In Proceedings of the 2nd ACM Symposium on Computational Geometry, pages 169-177, 1986.

[4] L. P. Chew. There are planar graphs almost as good as the complete graph. Journal of Computer and System Sciences, 39:205-219, 1989. 
[5] L. P. Chew and R. L. Drysdale. Voronoi diagrams based on convex distance functions. In Proceedings of the 1st ACM Symposium on Computational Geometry, pages 235-244, 1985.

[6] G. Das and D. Joseph. Which triangulations approximate the complete graph? In Proceedings of the International Symposium on Optimal Algorithms, volume 401 of Lecture Notes in Computer Science, pages 168-192, Berlin, 1989. Springer-Verlag.

[7] M. de Berg, M. van Kreveld, M. Overmars, and O. Schwarzkopf. Computational Geometry: Algorithms and Applications. Springer-Verlag, Berlin, 2nd edition, 2000.

[8] D. P. Dobkin, S. J. Friedman, and K. J. Supowit. Delaunay graphs are almost as good as complete graphs. Discrete \& Computational Geometry, 5:399-407, 1990.

[9] J. M. Keil and C. A. Gutwin. Classes of graphs which approximate the complete Euclidean graph. Discrete \& Computational Geometry, 7:13-28, 1992.

[10] R. Klein and D. Wood. Voronoi diagrams based on general metrics in the plane. In Proceedings of the 5th Symposium on Theoretical Aspects of Computer Science, volume 294 of Lecture Notes in Computer Science, pages 281-291, Berlin, 1988. SpringerVerlag.

[11] L. Ma. Bisectors and Voronoi Diagrams for Convex Distance Functions. Ph.D. thesis, Department of Computer Science, FernUniversität Hagen, Germany, 2000.

[12] G. Narasimhan and M. Smid. Geometric Spanner Networks. Cambridge University Press, Cambridge, UK, 2007. 